Supporting Information

\title{
A Joint Venture of Ab Initio Molecular Dynamics, Coupled Cluster Electronic Structure Methods, and Liquid State Theory to Compute Accurate Isotropic Hyperfine Constants of Nitroxide Probes in Water
}

Bikramjit Sharma, ${ }^{a}$ Van Anh Tran, ${ }^{\mathrm{b}}$ Tim Pongratz, ${ }^{\mathrm{c}}$ Laura Galazzo, ${ }^{\mathrm{d}}$ Irina Zhurko, ${ }^{\mathrm{e}}$

Enrica Bordignon, ${ }^{d *}$ Stefan M. Kast, ${ }^{c^{*}}$ Frank Neese,${ }^{\text {b* }}$ Dominik Marx ${ }^{a^{*}}$

a Lehrstuhl für Theoretische Chemie, Ruhr-Universität Bochum, 44780 Bochum, Germany

b Max-Planck-Institut für Kohlenforschung, Kaiser-Wilhelm-Platz 1, 45470 Mülheim an der Ruhr, Germany

c Physikalische Chemie III, Technische Universität Dortmund, Otto-Hahn-Str. 4a, 44227 Dortmund, Germany

d Faculty of Chemistry and Biochemistry, Ruhr-Universität Bochum, 44780 Bochum, Germany

e Laboratory of Nitrogen Compounds, N.N. Vorozhtsov Novosibirsk Institute of Organic Chemistry, NIOCH SB RAS, 9 Lavrentiev Avenue, 630090, Novosibirsk, Russia 


\section{Geometry optimization and Gibbs energies of HMI diastereomers}

Table S1. Relative Gibbs energies $G_{\text {sol }}$ (in kcal mol-1) and contributions (for EC-RISM: ${ }^{1}$ sum of intramolecular energy $E_{\text {sol }}$, excess chemical potential $\mu^{\mathrm{ex}}$, partial molar volume correction $c_{v} V_{m}$ with $c_{V}$ $=-0.1022 \mathrm{kcal} \mathrm{mol}^{-1} \AA^{-3}$ ) from calculations on optimized solution structures (Tables S2-S4).

\begin{tabular}{|c|c|c|c|c|c|}
\hline Method / Isomer & $E_{\text {sol }}$ & $\mu^{\mathrm{ex}}$ & $c_{v} V_{\mathrm{m}}$ & Gsol $($ EC-RISM) & $G_{\text {sol }}(\mathrm{CPCM})$ \\
\hline \multicolumn{6}{|c|}{ MP2/6-311+G(d,p)/EC-RISM//B3LYP/6-311+G(d,p)/CPCM } \\
\hline$(3 R, 4 S)$ & 0 & 0 & 0 & 0 & \\
\hline$(3 R, 4 R)$ & 5.39 & -1.46 & 0.30 & 4.23 & 4.65 \\
\hline \multicolumn{6}{|c|}{ MP2/6-311+G(d,p)/EC-RISM//B3LYP/def2-TZVPP/CPCM } \\
\hline$(3 R, 4 S)$ & 0 & 0 & 0 & 0 & \\
\hline$(3 R, 4 R)$ & 5.38 & -1.42 & 0.29 & 4.25 & 4.90 \\
\hline \multicolumn{6}{|c|}{ revPBE0-D3/def2tzvpp/EC-RISM//revPBE0-D3/def2tzvpp/CPCM } \\
\hline$(3 R, 4 S)$ & 0 & 0 & 0 & 0 & \\
\hline$(3 R, 4 R)$ & 4.95 & -1.63 & 0.32 & 3.64 & 4.44 \\
\hline
\end{tabular}

Table S2. Coordinates (in $\AA$ ) of optimized solution structures (B3LYP/6-311+G(d,p)/CPCM).

\begin{tabular}{lrrrrrr}
\hline Atom / Isomer & $x$ & $y$ & \multicolumn{3}{c}{$z$} & $y$ \\
\hline$(3 R, 4 S)$ & & \multicolumn{2}{c}{$(3 R, 4 R)$} & & \\
$\mathrm{N}$ & 8.252476 & 8.337704 & 7.426601 & 8.037483 & 8.90737 & 7.203353 \\
$\mathrm{C}$ & 7.319935 & 9.504649 & 7.635594 & 7.203373 & 10.13494 & 7.310678 \\
$\mathrm{~N}$ & 7.823836 & 10.492200 & 6.669654 & 7.914692 & 11.01601 & 6.360682 \\
$\mathrm{C}$ & 9.289323 & 10.23990 & 6.567672 & 9.340434 & 10.63951 & 6.379136 \\
$\mathrm{C}$ & 9.492319 & 8.696310 & 6.681296 & 9.298843 & 9.091462 & 6.438623 \\
$\mathrm{O}$ & 8.092050 & 7.214203 & 8.009302 & 7.673226 & 7.789077 & 7.694933 \\
$\mathrm{C}$ & 9.509107 & 7.929554 & 5.348223 & 9.149610 & 8.410110 & 5.066547 \\
$\mathrm{C}$ & 10.73106 & 8.341047 & 7.512287 & 10.48055 & 8.499844 & 7.211611 \\
$\mathrm{H}$ & 8.657329 & 8.186530 & 4.715495 & 8.354930 & 8.874042 & 4.478577 \\
$\mathrm{H}$ & 9.476532 & 6.855247 & 5.549162 & 8.908816 & 7.353909 & 5.210471 \\
$\mathrm{H}$ & 10.42671 & 8.143521 & 4.794143 & 10.08452 & 8.473281 & 4.505387 \\
$\mathrm{H}$ & 11.63179 & 8.679804 & 6.992909 & 11.41231 & 8.719636 & 6.683294 \\
$\mathrm{H}$ & 10.80478 & 7.260596 & 7.653049 & 10.38293 & 7.415113 & 7.290962 \\
$\mathrm{H}$ & 10.69975 & 8.820097 & 8.493430 & 10.54571 & 8.920110 & 8.217941 \\
$\mathrm{C}$ & 5.878591 & 9.045851 & 7.418225 & 5.775957 & 9.851241 & 6.837061 \\
$\mathrm{C}$ & 7.486529 & 10.01918 & 9.079758 & 7.210928 & 10.60304 & 8.781233 \\
$\mathrm{H}$ & 5.731616 & 8.603855 & 6.432241 & 5.785080 & 9.453963 & 5.820387 \\
$\mathrm{H}$ & 5.194091 & 9.888055 & 7.538599 & 5.189311 & 10.77257 & 6.848138 \\
$\mathrm{H}$ & 8.490757 & 10.40685 & 9.259847 & 5.290619 & 9.130518 & 7.498468 \\
$\mathrm{H}$ & 7.293350 & 9.212604 & 9.791272 & 8.216148 & 10.87735 & 9.109576 \\
$\mathrm{H}$ & 6.768435 & 10.82344 & 9.255861 & 6.847116 & 9.794085 & 9.418564 \\
$\mathrm{C}$ & 9.980685 & 10.91229 & 5.388939 & 6.551276 & 11.46342 & 8.912676 \\
$\mathrm{H}$ & 9.638396 & 10.54012 & 4.422015 & 10.12790 & 11.22461 & 5.210081 \\
$\mathrm{H}$ & 11.05805 & 10.73699 & 5.455200 & 9.646917 & 11.00451 & 4.254008 \\
$\mathrm{H}$ & 9.817616 & 11.99278 & 5.420828 & 11.14021 & 10.81177 & 5.194528 \\
$\mathrm{C}$ & 7.085344 & 10.63170 & 5.408797 & 10.22031 & 12.30814 & 5.307538 \\
$\mathrm{H}$ & 7.045121 & 9.729017 & 4.782137 & 7.661777 & 12.44553 & 6.523109 \\
$\mathrm{H}$ & 7.539299 & 11.43054 & 4.822606 & 8.119968 & 12.86920 & 7.431049 \\
$\mathrm{H}$ & 6.061427 & 10.93870 & 5.624625 & 6.586739 & 12.63096 & 6.560007 \\
$\mathrm{H}$ & 9.731190 & 10.66633 & 7.474085 & 8.058373 & 12.98481 & 5.661688 \\
$\mathrm{H}$ & 5.633463 & 8.295706 & 8.171597 & 9.814108 & 10.99061 & 7.313403 \\
\hline
\end{tabular}


Table S3. Coordinates (in $\AA$ ) of optimized solution structures (B3LYP/def2-TZVPP/CPCM).

\begin{tabular}{|c|c|c|c|c|c|c|}
\hline Atom / Isomer & $x$ & $y$ & $z$ & $x$ & $y$ & \\
\hline$(3 R, 4 S)$ & \multicolumn{6}{|c|}{$(3 R, 4 R)$} \\
\hline $\mathrm{N}$ & 8.256232 & 8.341695 & 7.430327 & 8.038651 & 8.910431 & 7.201565 \\
\hline C & 7.324564 & 9.503769 & 7.632533 & 7.208654 & 10.13454 & 7.310235 \\
\hline $\mathrm{N}$ & 7.827026 & 10.48725 & 6.667828 & 7.918481 & 11.01499 & 6.365774 \\
\hline C & 9.288164 & 10.23748 & 6.567218 & 9.33986 & 10.63933 & 6.380678 \\
\hline C & 9.48926 & 8.696947 & 6.682638 & 9.296449 & 9.094792 & 6.440155 \\
\hline $\mathrm{O}$ & 8.094922 & 7.220640 & 8.009366 & 7.673964 & 7.793967 & 7.687767 \\
\hline C & 9.505257 & 7.929457 & 5.353988 & 9.149247 & 8.415227 & 5.070895 \\
\hline C & 10.72743 & 8.342484 & 7.508435 & 10.47465 & 8.502081 & 7.210723 \\
\hline $\mathrm{H}$ & 8.658454 & 8.187979 & 4.719426 & 8.359607 & 8.880868 & 4.482023 \\
\hline $\mathrm{H}$ & 9.465505 & 6.858282 & 5.557322 & 8.90345 & 7.362685 & 5.214451 \\
\hline $\mathrm{H}$ & 10.42305 & 8.135385 & 4.802272 & 10.08325 & 8.473396 & 4.512614 \\
\hline $\mathrm{H}$ & 11.62610 & 8.675155 & 6.987045 & 11.40465 & 8.713033 & 6.680968 \\
\hline $\mathrm{H}$ & 10.79831 & 7.265055 & 7.654586 & 10.37126 & 7.420783 & 7.296105 \\
\hline $\mathrm{H}$ & 10.70049 & 8.824799 & 8.485412 & 10.54469 & 8.925323 & 8.212840 \\
\hline C & 5.887422 & 9.043122 & 7.415080 & 5.783618 & 9.853491 & 6.838199 \\
\hline C & 7.484424 & 10.02014 & 9.073272 & 7.213512 & 10.59971 & 8.778239 \\
\hline $\mathrm{H}$ & 5.740025 & 8.608601 & 6.428498 & 5.790725 & 9.466121 & 5.820355 \\
\hline $\mathrm{H}$ & 5.201090 & 9.879538 & 7.542339 & 5.196272 & 10.77120 & 6.858238 \\
\hline $\mathrm{H}$ & 8.484641 & 10.41095 & 9.254386 & 5.301957 & 9.127418 & 7.492177 \\
\hline $\mathrm{H}$ & 7.294214 & 9.215536 & 9.784024 & 8.215219 & 10.87678 & 9.106861 \\
\hline $\mathrm{H}$ & 6.764847 & 10.81998 & 9.248005 & 6.853634 & 9.790743 & 9.413493 \\
\hline C & 9.978080 & 10.90801 & 5.391721 & 6.552786 & 11.45602 & 8.910331 \\
\hline $\mathrm{H}$ & 9.635943 & 10.53733 & 4.426952 & 10.12388 & 11.22275 & 5.213407 \\
\hline $\mathrm{H}$ & 11.05309 & 10.73337 & 5.456771 & 9.643763 & 11.00256 & 4.259742 \\
\hline $\mathrm{H}$ & 9.814938 & 11.98605 & 5.422659 & 11.13425 & 10.81177 & 5.196778 \\
\hline C & 7.088366 & 10.63643 & 5.414155 & 10.21539 & 12.30405 & 5.309200 \\
\hline $\mathrm{H}$ & 7.045128 & 9.741285 & 4.780618 & 7.661417 & 12.43951 & 6.519974 \\
\hline $\mathrm{H}$ & 7.539966 & 11.43715 & 4.832957 & 8.112958 & 12.86962 & 7.425601 \\
\hline $\mathrm{H}$ & 6.066878 & 10.94120 & 5.632551 & 6.588071 & 12.62194 & 6.552933 \\
\hline $\mathrm{H}$ & 9.730093 & 10.66245 & 7.471834 & 8.058513 & 12.9767 & 5.660365 \\
\hline$\underline{H}$ & 5.647057 & 8.287866 & 8.161234 & 9.817339 & 10.98892 & 7.311024 \\
\hline
\end{tabular}

Table S4. Coordinates (in $\AA$ ) of optimized solution structures (revPBE0-D3/def2-TZVPP/CPCM).

\begin{tabular}{lrrrrrr}
\hline Atom / Isomer & $x$ & $y$ & $z$ & $x$ & $y$ \\
\hline$(3 R, 4 S)$ & & \multicolumn{5}{c}{$(3 R, 4 R)$} \\
N & 8.256232 & 8.341695 & 7.430327 & 8.038651 & 8.910431 & 7.201565 \\
$\mathrm{C}$ & 7.324564 & 9.503769 & 7.632533 & 7.208654 & 10.13454 & 7.310235 \\
$\mathrm{~N}$ & 7.827026 & 10.48725 & 6.667828 & 7.918481 & 11.01499 & 6.365774 \\
$\mathrm{C}$ & 9.288164 & 10.23748 & 6.567218 & 9.33986 & 10.63933 & 6.380678 \\
$\mathrm{C}$ & 9.48926 & 8.696947 & 6.682638 & 9.296449 & 9.094792 & 6.440155 \\
$\mathrm{O}$ & 8.094922 & 7.220640 & 8.009366 & 7.673964 & 7.793967 & 7.687767 \\
$\mathrm{C}$ & 9.505257 & 7.929457 & 5.353988 & 9.149247 & 8.415227 & 5.070895 \\
$\mathrm{C}$ & 10.72743 & 8.342484 & 7.508435 & 10.47465 & 8.502081 & 7.210723 \\
$\mathrm{H}$ & 8.658454 & 8.187979 & 4.719426 & 8.359607 & 8.880868 & 4.482023 \\
$\mathrm{H}$ & 9.465505 & 6.858282 & 5.557322 & 8.90345 & 7.362685 & 5.214451 \\
$\mathrm{H}$ & 10.42305 & 8.135385 & 4.802272 & 10.08325 & 8.473396 & 4.512614 \\
$\mathrm{H}$ & 11.62610 & 8.675155 & 6.987045 & 11.40465 & 8.713033 & 6.680968 \\
$\mathrm{H}$ & 10.79831 & 7.265055 & 7.654586 & 10.37126 & 7.420783 & 7.296105 \\
$\mathrm{H}$ & 10.70049 & 8.824799 & 8.485412 & 10.54469 & 8.925323 & 8.212840 \\
$\mathrm{C}$ & 5.887422 & 9.043122 & 7.415080 & 5.783618 & 9.853491 & 6.838199 \\
$\mathrm{C}$ & 7.484424 & 10.02014 & 9.073272 & 7.213512 & 10.59971 & 8.778239 \\
$\mathrm{H}$ & 5.740025 & 8.608601 & 6.428498 & 5.790725 & 9.466121 & 5.820355 \\
$\mathrm{H}$ & 5.201090 & 9.879538 & 7.542339 & 5.196272 & 10.77120 & 6.858238 \\
$\mathrm{H}$ & 8.484641 & 10.41095 & 9.254386 & 5.301957 & 9.127418 & 7.492177 \\
$\mathrm{H}$ & 7.294214 & 9.215536 & 9.784024 & 8.215219 & 10.87678 & 9.106861
\end{tabular}




\begin{tabular}{lrrrrrr}
\hline Atom / Isomer & $x$ & $y$ & $z$ & $x$ & $y$ & $z$ \\
\hline$(3 R, 4 S)$ & 8.256232 & 8.341695 & 7.430327 & 8.038651 & 8.910431 & 7.201565 \\
$\mathrm{~N}$ & 6.764847 & 10.81998 & 9.248005 & 6.853634 & 9.790743 & 9.413493 \\
$\mathrm{H}$ & 9.978080 & 10.90801 & 5.391721 & 6.552786 & 11.45602 & 8.910331 \\
$\mathrm{C}$ & 9.635943 & 10.53733 & 4.426952 & 10.12388 & 11.22275 & 5.213407 \\
$\mathrm{H}$ & 11.05309 & 10.73337 & 5.456771 & 9.643763 & 11.00256 & 4.259742 \\
$\mathrm{H}$ & 9.814938 & 11.98605 & 5.422659 & 11.13425 & 10.81177 & 5.196778 \\
$\mathrm{H}$ & 7.088366 & 10.63643 & 5.414155 & 10.21539 & 12.30405 & 5.309200 \\
$\mathrm{C}$ & 7.045128 & 9.741285 & 4.780618 & 7.661417 & 12.43951 & 6.519974 \\
$\mathrm{H}$ & 7.539966 & 11.43715 & 4.832957 & 8.112958 & 12.86962 & 7.425601 \\
$\mathrm{H}$ & 6.066878 & 10.94120 & 5.632551 & 6.588071 & 12.62194 & 6.552933 \\
$\mathrm{H}$ & 9.730093 & 10.66245 & 7.471834 & 8.058513 & 12.9767 & 5.660365 \\
$\mathrm{H}$ & 5.647057 & 8.287866 & 8.161234 & 9.817339 & 10.98892 & 7.311024 \\
$\mathrm{H}$ & & & & & &
\end{tabular}

Table S5. Coordinates (in $\AA$ ) of optimized $(3 R, 4 S$ ) vacuum structure (revPBE0-D3/def2-TZVPP).

\begin{tabular}{lrrr}
\hline Atom & $x$ & $y$ & $z$ \\
\hline $\mathrm{N}$ & 7.913225 & 6.621944 & 8.039936 \\
$\mathrm{C}$ & 7.711075 & 7.468328 & 9.228306 \\
$\mathrm{~N}$ & 7.704538 & 8.804437 & 8.620663 \\
$\mathrm{C}$ & 8.482493 & 8.752174 & 7.384042 \\
$\mathrm{C}$ & 8.121292 & 7.379223 & 6.795154 \\
$\mathrm{O}$ & 7.7901 & 5.365998 & 8.079218 \\
$\mathrm{C}$ & 6.82233 & 7.363257 & 5.989732 \\
$\mathrm{C}$ & 9.260144 & 6.766624 & 5.995318 \\
$\mathrm{H}$ & 6.530469 & 6.328791 & 5.813671 \\
$\mathrm{H}$ & 6.954408 & 7.851527 & 5.024873 \\
$\mathrm{H}$ & 6.021512 & 7.864289 & 6.530558 \\
$\mathrm{H}$ & 9.453605 & 7.362457 & 5.103531 \\
$\mathrm{H}$ & 9.000124 & 5.756176 & 5.686379 \\
$\mathrm{H}$ & 10.17307 & 6.718692 & 6.587418 \\
$\mathrm{C}$ & 6.363431 & 7.164709 & 9.867869 \\
$\mathrm{C}$ & 8.855246 & 7.184828 & 10.20591 \\
$\mathrm{H}$ & 5.564759 & 7.328934 & 9.148182 \\
$\mathrm{H}$ & 6.198016 & 7.816114 & 10.72522 \\
$\mathrm{H}$ & 6.334358 & 6.130405 & 10.20437 \\
$\mathrm{H}$ & 9.819671 & 7.458165 & 9.779399 \\
$\mathrm{H}$ & 8.867987 & 6.118233 & 10.42305 \\
$\mathrm{C}$ & 8.717537 & 7.724639 & 11.14144 \\
$\mathrm{H}$ & 8.199505 & 9.930989 & 6.471426 \\
$\mathrm{H}$ & 8.570622 & 10.86009 & 6.900564 \\
$\mathrm{H}$ & 7.131331 & 10.03922 & 6.29326 \\
$\mathrm{C}$ & 8.700863 & 9.797249 & 5.513098 \\
$\mathrm{H}$ & 8.06974 & 9.885717 & 9.510373 \\
$\mathrm{H}$ & 7.495546 & 9.818053 & 10.4327 \\
$\mathrm{H}$ & 7.825269 & 10.84088 & 9.050864 \\
$\mathrm{H}$ & 9.137995 & 9.893318 & 9.769603 \\
$\mathrm{H}$ & 9.563533 & 8.74098 & 7.605044 \\
\hline & & &
\end{tabular}

\section{Details of FFMD for preparing AIMD simulations}

Force field simulation of $\mathrm{HMI}$ in water

In order to prepare the initial conditions for the AIMD simulation, we carried out force field simulations to pre-equilibrate HMI in water. To this end, we have chosen the TIP4P-2005 water model ${ }^{2}$ for these FFMD simulations. For bulk water, this particular force field has been found to perform very well ${ }^{3}$ 
within the class of simple water models which are both non-polarizable and rigid. Since our goal is to determine the fixed box length to be used in the NVT AIMD simulations of HMI in water using FFMD and given that the density of this aqueous solution is largely determined by the density of the solvent itself, the TIP4P-2005 force field is an excellent choice since it reproduces the density of water in a large part of its phase diagram, notably including many ice phases.
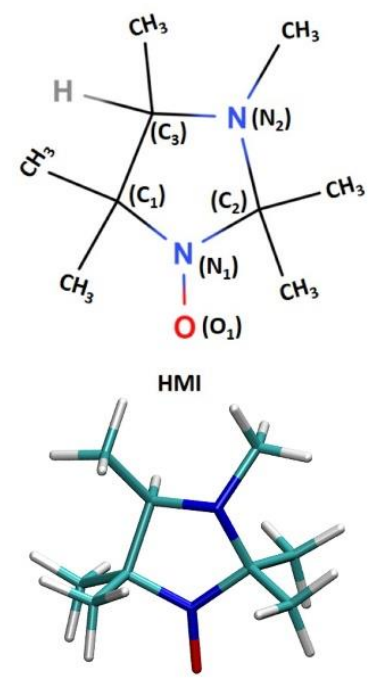

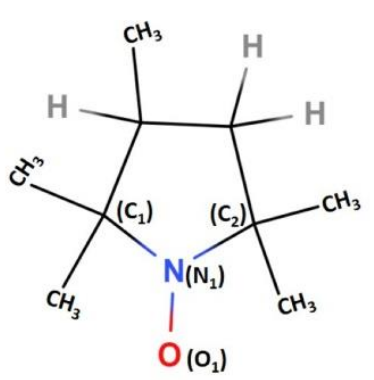

SLR

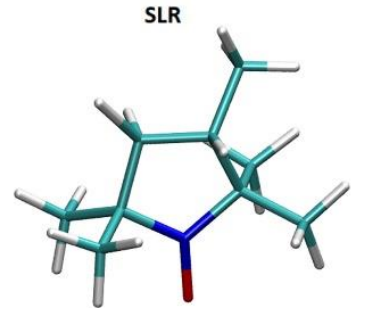<smiles>[2H][NH+]1CCCC1</smiles>

PROLINE

Fig. S1. Comparison of structures of HMI and SLR with proline.

Concerning the force field parameters of $\mathrm{HMI}$, we have borrowed parameters from two structurally similar molecules, namely proline and SLR (Fig. S1). SLR is an EPR probe and differs from HMI in having a carbon atom instead of the other nitrogen site in the ring. Thus, we have adopted parameters of SLR from Ref. ${ }^{4}$ and of proline from a recent CHARMM force field ${ }^{5}$ to represent similar atoms of HMI (see Table S5 for parameters). With these force field parameters, we have performed FFMD simulations in the NpT ensemble for 10 ns using the Gromacs simulation package. ${ }^{6-9}$ The pressure and temperature were kept at 1 atm and $300 \mathrm{~K}$ using the Parrinello-Rahman ${ }^{10}$ barostat and the canonical sampling through a velocity rescaling ${ }^{11}$ thermostat, respectively. The average box length of $15.9581 \AA$ obtained from this $N p T$ simulation (after equilibration) was taken to be the cubic box parameter for the subsequent runs as follows. A random configuration sampled at this average box length was used to continue the FFMD simulation in the NVT ensemble (using this fixed box length) for another $10 \mathrm{~ns}$. The final configuration from this $N V T$ run was taken to be the starting configuration for the AIMD simulation. We compare in Fig. S2 the average local solvation structure around the HMI oxygen obtained from FFMD and AIMD (after a sufficiently long trajectory was generated). The deviation of FFMD compared to the AIMD reference is in an acceptable range, thus supporting the use of FFMD for pre-equilibration. However, the differences are substantial enough to not rely on FFMD in order to sample the configuration snapshot ensembles for subsequent single-point property calculations.

Table S6. Atomic charges (in e) and atom types of HMI sites adopted for force field molecular dynamics simulations of $\mathrm{HMI}$ in water.

\begin{tabular}{lllr}
\hline HMI atom & SLR atom & Proline atom & Charge \\
\hline N1 & N1 & - & 0.249 \\
O1 & O1 & - & -0.433 \\
C1 & C1 & - & 0.245 \\
C2 & C2 & - & 0.267 \\
N2 & - & N2 & -0.290 \\
C3 & - & - & -0.038 \\
Methyl C & - & - & -0.27 \\
Methyl H & - & - & 0.09 \\
H bonded to C3 & - & - & 0.0 \\
\hline
\end{tabular}




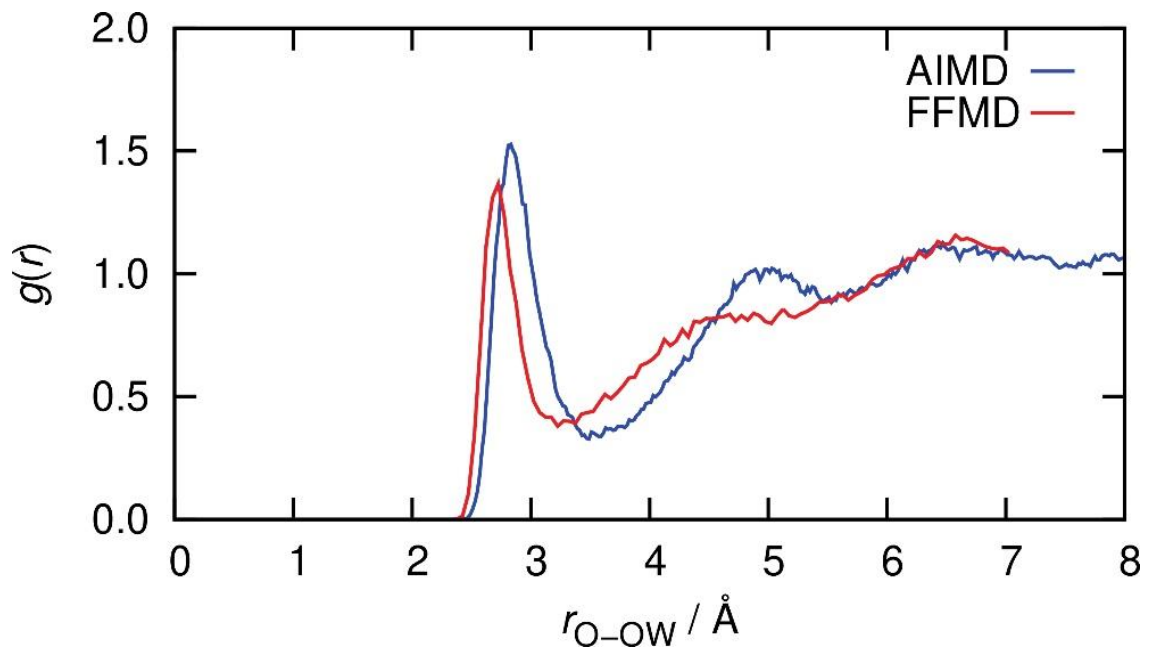

Fig. S2. Comparison of radial distribution functions for water oxygen (OW) with respect to HMl oxygen (O) obtained from AIMD and FFMD.

\section{Lennard-Jones parameters for EC-RISM calculations}

Table S7. Lennard-Jones parameters $\sigma$ (in $\AA$ ) and $\varepsilon$ (in $\mathrm{kJ} \mathrm{mol}^{-1}$ ) of atoms used in EC-RISM calculations. The numbers in parentheses refer to the corresponding GAFF-type atoms. ${ }^{12}$

\begin{tabular}{lrr}
\hline Atom & $\sigma$ & $\varepsilon$ \\
\hline $\mathrm{N}(3)$ & 3.249999 & 1.181109 \\
$\mathrm{C}(3)$ & 3.39967 & 0.760078 \\
$\mathrm{O}$ & 2.959922 & 1.459017 \\
$\mathrm{H}(\mathrm{C})$ & 2.649533 & 0.109079 \\
$\mathrm{H}(1)$ & 2.471353 & 0.109079 \\
\hline
\end{tabular}

\section{Representative snapshots of different QM/MM models}

A

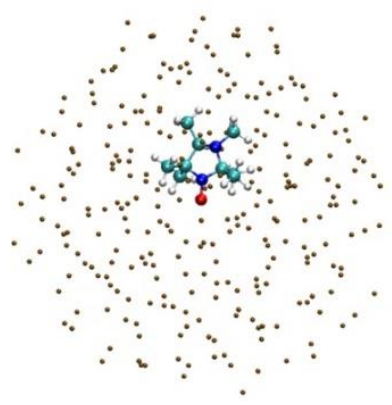

Only $\mathrm{HMI}$ in quantum region + all solvent atoms as TIP3P point charges
B

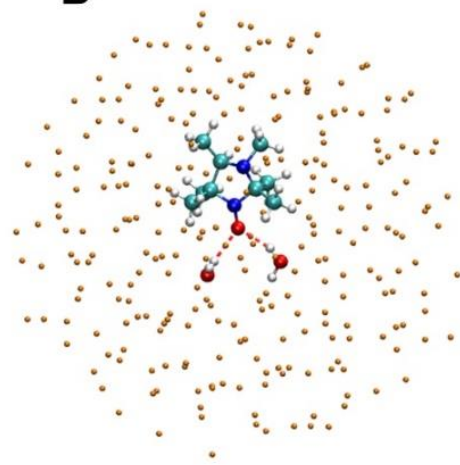

$\mathrm{HMI}$ and water molecules up to $1^{\text {st }}$ solvation shell in quantum region + remaining solvent atoms as TIP3P point charges

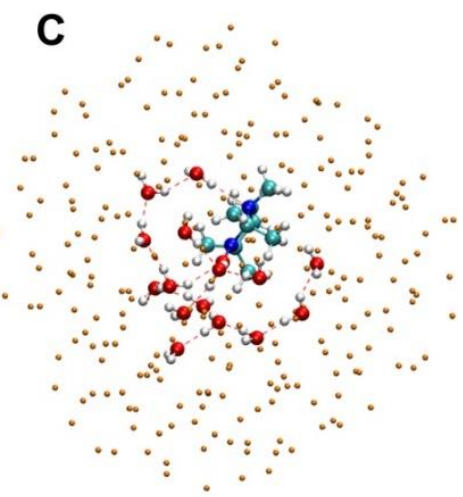

$\mathrm{HMI}$ and water molecules up to $2^{\text {nd }}$ solvation shell in quantum region + remaining solvent atoms as TIP3P point charges

Fig. S3. Representative snapshots of different QM/MM models examined in Section 5.2 of the main text. The dots represent the atoms of all water molecules beyond the QM region which are treated as TIP3P point charges in the MM embedding of the QM subsystem. (A) HMI with no solvation shell considered, (B) $\mathrm{HMI}_{+} 1^{\text {st }}$ solvation shell, and $(\mathrm{C}) \mathrm{HMI}+2^{\text {nd }}$ solvation shell as named in Table 5 of the main text. 


\section{Comparison of $A^{\text {iso }}$ distributions for nitroxy oxygen from DFT}
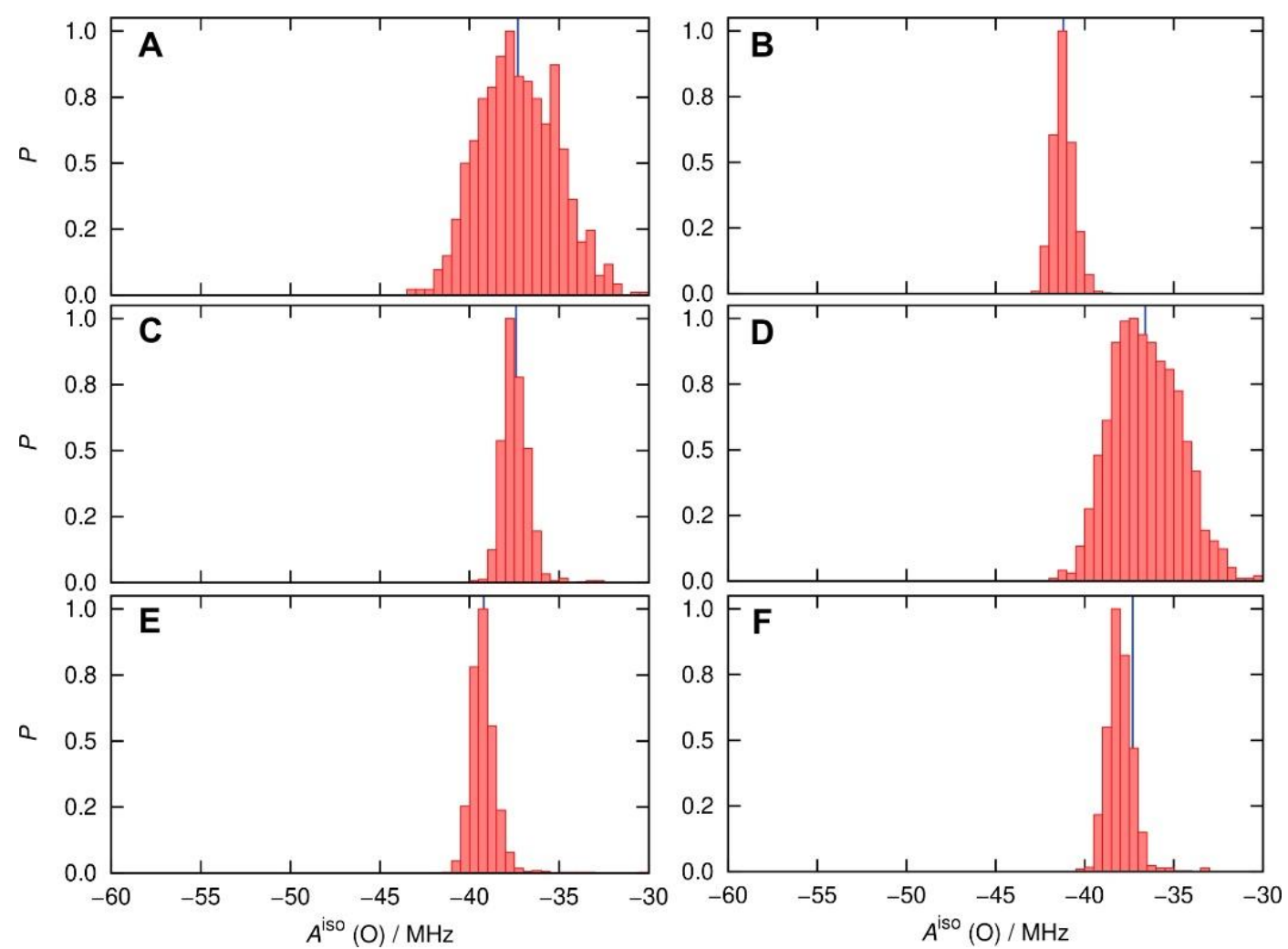

Fig. S4. Probability distributions (normalized by setting the respective maximum bin values to 1) of nitroxy oxygen $A^{\text {iso }}$ values calculated with revPBE0-D3 and s-contracted def2-TZVPP basis set; (A) $\mathrm{HMI}$ with explicit water, $(\mathrm{B})$ vertically desolvated $\mathrm{HMI},(\mathrm{C})$ vertically desolvated HMI with EC-RISM solvation, (D) vertically desolvated $\mathrm{HMI}$ with water molecules up to second solvation shell of $\mathrm{HMI}$ oxygen with EC-RISM solvation, $(E)$ vertically desolvated HMI with CPCM solvation, $(F)$ vertically desolvated HMI with water molecules up to second solvation shell of HMI oxygen with CPCM solvation. The blue line in each figure represents the average value of the corresponding distribution.

\section{Influence of different EC-RISM electrostatics models}
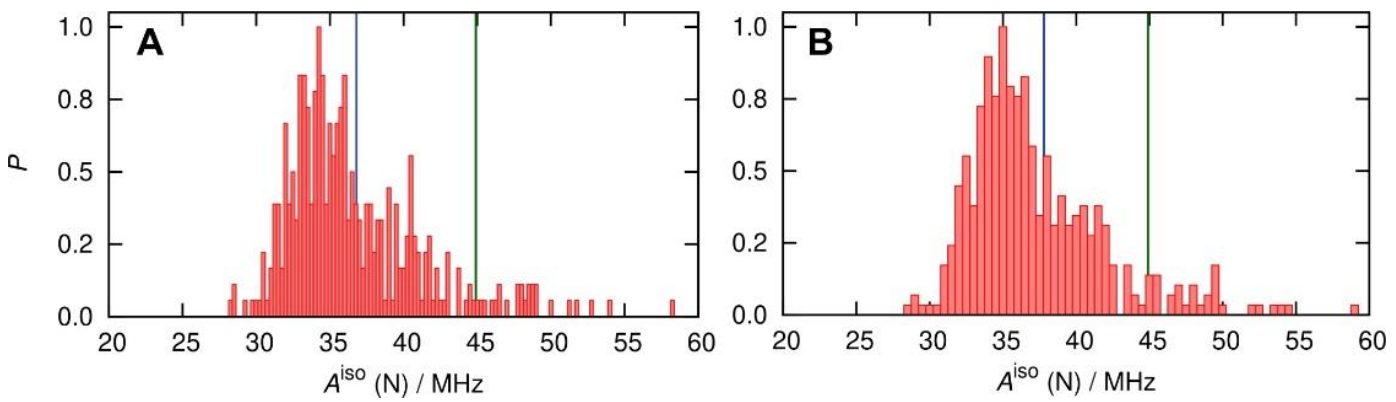

Fig. S5. Probability distributions (normalized by setting the respective maximum bin values to 1) of $A^{\text {iso }}$ values of nitroxy nitrogen calculated with revPBE0-D3 and s-contracted def2-TZVPP basis set using the AIMD ensemble of vertically desolvated spin probe configurations; $(A)$ with the consistent DFT electron density to calculate the exact electrostatic potential, $(B)$ with exact electrostatics from the HF electron density, as used within DLPNO-CCSD/def2-TZVPP. The blue line in each figure represents the average value of corresponding distribution. The green vertical lines represent the experimental benchmark value of $44.87 \pm 0.14 \mathrm{MHz}$. 


\section{References}

1. Tielker, N.; Eberlein, L.; Güssregen, S.; Kast, S. M. The SAMPL6 Challenge on Predicting Aqueous $p K_{a}$ Values from EC-RISM Theory. J. Comput. Aid. Mol. Des. 2018, 32, 1151-1163.

2. Abascal, J. L. F.; Vega C. A General Purpose Model for the Condensed Phases of Water: TIP4P/2005. J. Chem. Phys. 2005, 123, 234505.

3. Vega, C.; Abascal J. L. F.; Simulating Water with Rigid Non-polarizable Models: A General Perspective. Phys. Chem. Chem. Phys. 2011, 13, 19663-19688.

4. Sezer, D., Freed, J. H., Roux, B. Parametrization, Molecular Dynamics Simulation, and Calculation of Electron Spin Resonance Spectra of a Nitroxide Spin Label on a Polyalanine a-helix. J. Phys. Chem. B. 2008, 112, 5755-5767.

5. Huang, J.; Rauscher, S.; Nawrocki, G.; Ran, T.; Feig, M.; de Groot B. L. et al. CHARMM36m: An Improved Force Field for Folded and Intrinsically Disordered Proteins. Nat. Methods 2017, 14, 71-73.

6. Pronk, S.; Páll, S.; Schulz, R.; Larsson, P.; Bjelkmar, P.; Apostolov, R.; et al. GROMACS 4.5: A HighThroughput and Highly Parallel Open Source Molecular Simulation Toolkit. Bioinformatics 2013, 29, 845-854.

7. van der Spoel, D.; Lindahl, E.; Hess, B.; Groenhof, G.; Mark, A. E.; Berendsen, H. J. C. GROMACS: Fast, Flexible, and Free. J. Comput. Chem. 2005, 26, 1701-1718.

8. Hess, B.; Kutzner, C.; van der Spoel, D.; Lindahl, E.; GROMACS 4: Algorithms for Highly Efficient, Load-balanced, and Scalable Molecular Simulation. J. Chem. Theory Comput. 2008, 4, 435-447.

9. Abraham, M. J.; van der Spoel, D.; Lindahl, E.; Hess, B., and the G development team, GROMACS User Manual version 2019, https://www.gromacs.org.

10. Parrinello, M.; Rahman, A. Polymorphic Transitions in Single Crystals: A New Molecular Dynamics Method. J. Appl. Phys. 1981, 52, 7182-90.

11. Bussi, G.; Donadio, D.; Parrinello, M. Canonical Sampling Through Velocity Rescaling. J. Chem. Phys. 2007, 126, 014101.

12. Wang, J.; Wolf, R. M.; Caldwell, J. W.; Kollman, P. A.; Case, D. A. Development and Testing of a General Amber Force Field. J. Comput. Chem. 2004, 25, 1157-1174. 